\title{
Destinação da Fauna Silvestre Resgatada pela Equipe da Reserva Biológica Guaribas na Mata Atlântica do Nordeste Brasileiro
}

\author{
Talis Brito da Silva ${ }^{1} \&$ Afonso Henrique Leal ${ }^{2}$
}

Recebido em 20/07/2020 - Aceito em 13/08/2020

\author{
${ }^{1}$ Universidade Federal da Paraíba/UFPB, Brasil. <tallis.brito@hotmail.com> \\ ${ }^{2}$ Instituto Chico Mendes de Conservação da Biodiversidade/ICMBio, Brasil. < afonso.leal@icmbio.gov.br>.
}

\begin{abstract}
RESUMO - O objetivo deste trabalho é avaliar a destinação dada pela equipe da Reserva Biológica (REBIO) Guaribas aos animais silvestres por ela resgatados ou recebidos de resgate. Foram compiladas informações documentais, a fim de se identificar o perfil taxonômico da fauna resgatada, o perfil dos resgatadores, o tipo de destinação dada a ela e detectar tendências espaciais das solturas. Foram examinados 360 documentos do tipo "registro de fauna" produzidos entre abril de 2012 até julho de 2017, dos quais 114 caracterizam-se como eventos de resgates (31,7\% dos registros) e, destes, 31 registros ( $27 \%$ dos resgates) possibilitaram uma análise espacial. Mammalia foi o grupo taxonômico com maior representatividade, com destaque para a preguiça-comum (Bradypus variegatus). Detectou-se a oportunidade de aproveitar parte dos indivíduos dessa espécie, resgatados próximo à SEMA 2, em um programa de revigoramento populacional a ser criado na área da SEMA 3. Os tipos de resgatador mais frequentes foram o cidadão e a equipe da REBIO Guaribas, com 52 resgates $(45,6 \%)$ cada um. Observou-se que a destinação mais comum foi a soltura em ambiente natural, mas há uma elevada proporção de indivíduos resgatados feridos a ponto de serem encaminhados a cuidados veterinários ou irem a óbito. A equipe poderia melhorar a qualidade dos registros por meio da capacitação permanente dos executores das ações de manejo, incorporação de voluntários, bem como a melhoria da qualidade das solturas por meio de parceria com instituição de pesquisa.
\end{abstract}

Palavras-chave: Manejo de fauna; animais silvestres; impactos antrópicos; área protegida; Floresta Atlântica.

\section{Destination of Wildlife Rescued by Reserva Biológica Guaribas Team, in the Atlantic Forest of Northeastern Brazil}

\begin{abstract}
The aim of this work is to evaluate the destination given by the Reserva Biológica (REBIO) Guaribas team to wild animals rescued or received by them. Documentary information was compiled to identify the taxonomic profile of the rescued fauna, the profile of the rescuers, the type of destination given to it and to detect spatial trends in the releases. 360 documents of the type "fauna record" produced between April 2012 and July 2017 were examined, of which 114 were characterized as rescue events (31.7\% of the records) and of these, 31 records (27\% of the rescues) enabled a spatial analysis. Mammalia was the most represented taxonomic group, with emphasis on the common sloth (Bradypus variegatus). An opportunity was detected to use of part of the individuals of this species, rescued near SEMA 2 in a population reinvigoration program to be created in the SEMA 3 area. The most frequent types of rescuer were the citizen and the staff of REBIO Guaribas, with 52 rescues $(45.6 \%)$ each. The most common destination was release in a natural environment, but there is a high proportion of rescued individuals injured therefore being referred to veterinary care or dying. The team could improve the quality of the records through the permanent training of the executors of the management actions, incorporation of volunteers, as well improving as the quality of the releases through a partnership with a research institution.
\end{abstract}

Keywords: Wildlife management; wild animals; human impact; biological reserve; Atlantic Forest. 


\section{Destinación de la Fauna Silvestre Rescatada por el Personal de la Reserva Biologica Guaribas, en el Bosque Atlántico del Nordeste Brasileño}

RESUMEN - El objetivo de este trabajo es evaluar el destino dado por el equipo de la Reserva Biológica Guaribas (REBIO) a los animales salvajes rescatados o recibidos por ellos. Se recopiló información documental con el fin de identificar el perfil taxonómico de la fauna rescatada, el perfil de los rescatadores, el tipo de destino que se le dio y detectar tendencias espaciales en las liberaciones. Se examinaron 360 documentos del tipo de registro de fauna producido entre abril de 2012 y julio de 2017, de los cuales 114 fueron caracterizados como eventos de rescate (31,7\% de los registros) y de estos, 31 registros (27\% de los rescates) permitieron un análisis espacial. Mammalia fue el grupo taxonómico más representado, con énfasis en el perezoso común (Bradypus variegatus). Se detectó la oportunidad de aprovechar parte de los individuos de esta especie, rescatados cerca de SEMA 2 en un programa de revitalización poblacional que se creará en el área de SEMA 3. Los tipos de rescatadores más frecuentes fueron el ciudadano y el personal de REBIO Guaribas, con 52 rescates $(45,6 \%)$ cada uno. Se observó que el destino más común fue la liberación en un ambiente natural, pero existe una alta proporción de individuos rescatados lesionados al punto de ser referidos a atención veterinaria o fallecidos. El equipo podría mejorar la calidad de los registros a través de la capacitación permanente de los ejecutores de las acciones de manejo, incorporación de voluntarios, así como la mejoría de la calidad de las liberaciones mediante una asociación con una institución de investigación.

Palabras clave: Manejo de fauna; animales silvestres; área protegida; impactos antrópicos; Bosque Atlántico.

\section{Introdução}

O Brasil comporta um dos maiores contingentes de espécies silvestres do planeta e situa-se entre os maiores países do mundo em biodiversidade. Apesar desta posição privilegiada, o que se tem constatado é o rápido declínio das populações animais e o crescente risco de extinção de espécies em decorrência da perda e da fragmentação de habitat e da crescente ocupação humana e exploração econômica (Branco, 2002).

A pressão antrópica vem causando mudanças no habitat dos animais silvestres, acarretando o deslocamento desses animais que vão em busca de recursos ou deslocando-se por motivos diversos. Devido a isso, há um aumento no aparecimento deles em áreas urbanas (Pedrosa et al., 2016), e o resgate desses animais silvestres é comumente praticado para que não haja acidentes entre fauna e a população. $O$ termo resgate de fauna pode ser definido como "captura ou recolhimento, por autoridades competentes, de animais silvestres de vida livre em situação de risco ou que estejam em conflito com a população humana" (IBAMA, 2014). Essa atividade é rotina para quem atua na interface entre áreas silvestres $e$ antrópicas, como é o caso de muitas unidades de conservação, entre elas a Reserva Biológica (REBIO) Guaribas, localizada na Mata Atlântica Paraibana (Fig. 1).
Quando o destino do animal resgatado é a soltura, pode-se considerar que foi feita uma translocação, cuja definição é "o movimento mediado pelo ser humano de um organismo vivo a partir de uma área, com liberação em outra" (IUCN, 2013, tradução nossa). As translocações, de acordo com suas características e objetivos, podem ser de diferentes tipos e subtipos (IUCN/ SSC, 2013), dos quais Marini \& Marinho-Filho (2006) destacam três: introdução, reintrodução e revigoramento populacional. O primeiro é a liberação, intencional ou não, de um organismo fora da área de distribuição geográfica de sua espécie $e$ dificilmente tem objetivo conservacionista. O segundo é sua liberação intencional em uma área onde sua espécie tenha sido extinta localmente, seja por atividades humanas ou catástrofes naturais. O último é a liberação do organismo onde há uma população de sua espécie para melhorar a viabilidade populacional, aumentando a densidade, variabilidade genética ou equilibrando a razão sexual, por exemplo.

É comum a equipe do ICMBio, atuando na REBIO Guaribas, ser chamada para resgatar animais silvestres ou recebê-los quando são trazidos por pessoas da comunidade ou viajantes. Para a destinação, a gestão da unidade de conservação elaborou critérios semelhantes aos da norma do IBAMA (2014), segundo a seguinte chave de decisão: (1) encontrado morto ou veio a óbito após o resgate: doação para coleção científica da Universidade Federal da Paraíba - Campus I; 
(2) doente, ferido ou muito jovem, dependente de cuidado: cativeiro e (3) saudável e independente, a partir de avaliação do aspecto físico e do comportamento: soltura no fragmento florestal mais próximo de onde foi encontrado, com habitat adequado para a espécie. Este pode localizar-se na própria $\mathrm{REBIO}$ Guaribas ou em propriedades rurais vizinhas.

Antes do estabelecimento desse critério para as solturas, em 2010, todas eram feitas dentro da unidade de conservação, próximo à sua sede e, talvez, essa ainda seja uma tendência, apesar das novas orientações. Os destinos de cativeiro podem ser o CETAS/IBAMA da Paraíba ou o Parque Zoobotânico Arruda Câmara de João Pessoa. Nesses locais, os animais feridos, doentes ou muito jovens são reabilitados para uma soltura na natureza após um período mais longo, ou permanecerem em cativeiro até o fim de suas vidas, se a primeira opção não for viável. A destinação também é anotada no Registro de Fauna e, no caso de soltura, que é feita imediatamente ou, no máximo, no dia seguinte, são registradas as coordenadas geográficas do local.

Nesse contexto, o presente trabalho tem como objetivo geral avaliar a destinação dada, pela equipe da REBIO Guaribas, aos animais silvestres por ela resgatados ou recebidos, com ênfase nas solturas. Especificamente, ele visa identificar o perfil taxonômico da fauna resgatada $e$ o perfil dos resgatadores, e do tipo de destinação dada a ela. Quanto às solturas, verificar se elas apresentam tendências espaciais de aproximação ou afastamento da REBIO Guaribas, concentração em pontos específicos e retorno ao provável ambiente de origem.

\section{Material e Métodos \\ Área de estudo}

A REBIO Guaribas, localizada na região do litoral norte da Paraíba, protege 4.028,55 ha de florestas e formações savânicas na porção norte do bioma Mata Atlântica. Ela é formada por três áreas disjuntas denominadas, SEMA 1 e SEMA 2, no município de Mamanguape, e SEMA 3, no de rio Tinto, com 673,64ha, 3.016,09ha e 338,82ha, respectivamente (IBAMA, 2003). Atualmente, a REBIO Guaribas faz parte do Núcleo de Gestão Integrada ICMBio Mamanguape, juntamente com a Área de Proteção Ambiental (APA) da Barra do Rio Mamanguape e a Área de Relevante Interesse Ecológico (ARIE) dos Manguezais da Foz do Rio Mamanguape, situadas também no Litoral Norte da Paraíba (Fig. 1).

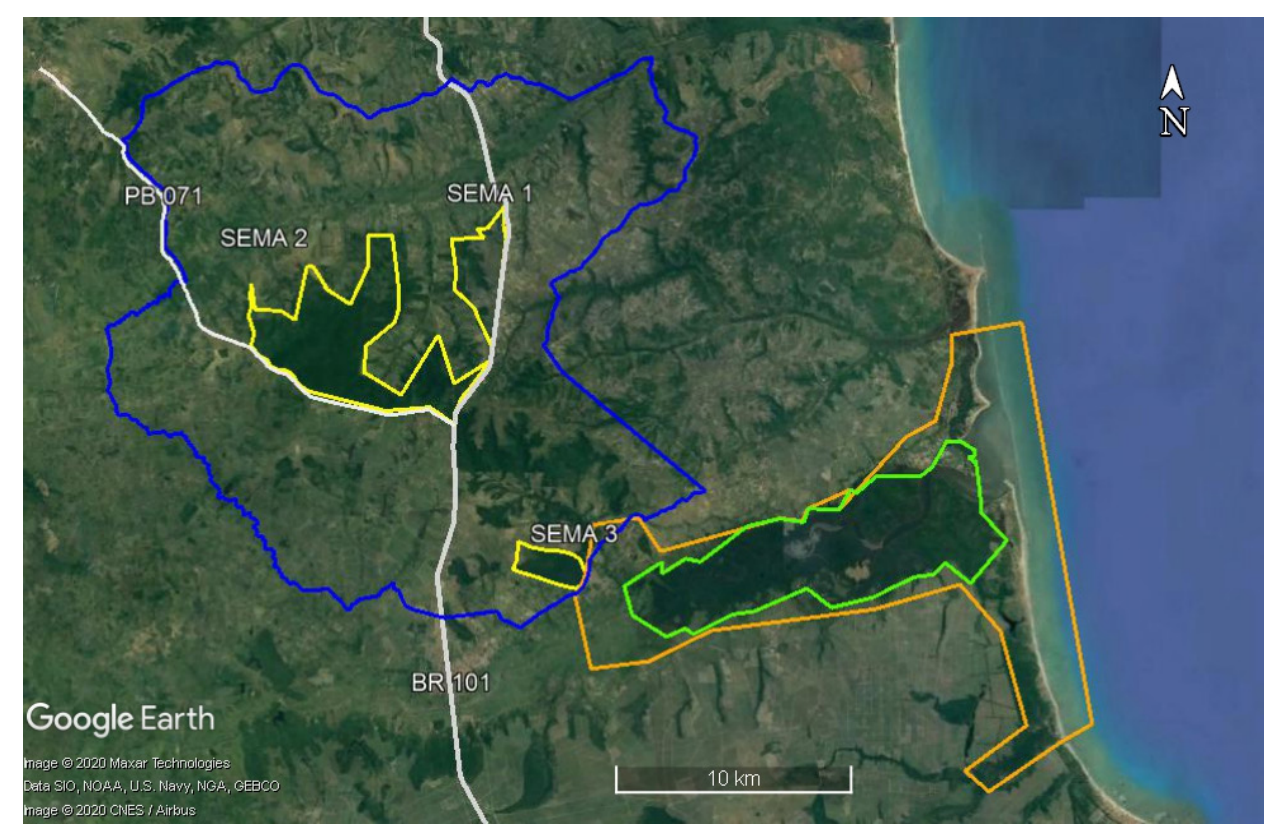

Figura 1 - Imagem de satélite do litoral norte da Paraíba, onde se situa a Reserva Biológica Guaribas (linhas amarelas). Também estão representadas sua zona de amortecimento (linha azul), a Área de Proteção Ambiental da Barra do Rio Mamanguape (linha laranja), a Área de Relevante Interesse Ecológico dos Manguezais da Foz do Rio Mamanguape (linha verde) e as rodovias BR 101 e PB 071 (linhas cinza). 


\section{Coleta e análise de dados}

Os dados foram coletados a partir de registros na REBIO Guaribas e áreas adjacentes, até onde há dados de vertebrados silvestres resgatados ou soltos pela equipe de profissionais da reserva.

Foram examinados todos os registros de fauna arquivados na REBIO Guaribas, desde que começaram a ser produzidos, em abril de 2012, até julho de 2017, e foram considerados para estudo aqueles que indicam eventos de resgate. Foi adotada a definição de resgate da Instrução Normativa IBAMA $\mathrm{n}^{\circ} 23$ de 2014 (IBAMA, 2014), citada anteriormente, estendendo esse entendimento também para quando o cidadão comum, e não apenas uma autoridade, efetua a captura e entrega o animal. De acordo com nosso entendimento, são exemplos de situação de risco ou de conflito com a população humana o animal estar presente em rodovias, áreas urbanas, habitações humanas, construções para animais domésticos e plantios em época de colheita ou aragem.

Dos registros considerados, foram compilados os seguintes dados em uma planilha do Microsoft Excel: espécie do animal, táxon superior, resgatador (equipe da REBIO, cidadão comum ou outra autoridade), data do resgate, da soltura, tipo de destinação (coleção, cativeiro ou soltura), coordenadas geográficas do local de resgate e do local de soltura e observações pertinentes para registro interno. Quando necessário, foram consultadas fotografias armazenadas dos animais resgatados para confirmar ou dar mais precisão à identificação da espécie. Quando não foram especificadas as coordenadas do local de resgate ou de soltura, a localidade descrita foi localizada na imagem de satélite gerada pelo software Google Earth Pro, de onde as coordenadas foram extraídas.

A partir da compilação dos dados, foram contadas as células em que estão presentes cada espécie e cada táxon superior, de modo a se conhecer a composição taxonômica do conjunto de animais resgatados e recebidos, a ser expressa em números absolutos e porcentagens. Da mesma forma, foram tratados os dados de tipo de destinação para se obter o perfil dessa atividade.

Para cada registro com soltura, foram marcados os pontos de resgate e de soltura na imagem do Google Earth Pro, o que possibilitou dois tipos de avaliação.
A primeira é se as escolhas dos locais de soltura estão seguindo o critério estabelecido pela gestão da REBIO. Para isso, em cada caso, é verificado se a soltura foi feita no fragmento florestal mais próximo do local de resgate $e$ assinalado "sim" ou "não" na planilha. O número de respostas "sim" foi contado para medir o sucesso em seguir tal critério.

A segunda avaliação é se as solturas estão trazendo os animais resgatados mais para próximo da REBIO, levando para mais longe ou os mantendo na distância original. Para tanto, foi medida a distância do ponto de resgate ao limite mais próximo da REBIO (Xr) e o equivalente para o ponto de soltura (Xs). Depois foi calculada a diferença (Xs-Xr), em que valores negativos indicam que o indivíduo foi trazido para mais próximo da REBIO. A média dessa diferença foi para se detectar uma tendência geral ao afastamento ou à aproximação à REBIO.

Para examinar visualmente essas tendências espaciais, um mapa de resgates e de solturas de fauna foi elaborado usando o programa Quantum GIS 1.14 (QGIS Development Team, 2016), as mesmas coordenadas citadas e o shapefile da REBIO Guaribas fornecido pelo ICMBio. Os registros da espécie ameaçada de primata guariba (Alouatta belzebul) não fizeram parte da análise espacial, pois as solturas de seus indivíduos são realizadas por critérios e decisão do Centro Nacional de Pesquisa e Conservação de Primatas Brasileiros (CPB) (Valença-Montenegro et al., 2018).

\section{Resultados e Discussão}

Foram examinados 360 registros de fauna produzidos entre abril de 2012 até julho de 2017, dos quais 114 caracterizaram-se como eventos de resgates, correspondendo a $31,7 \%$ do total de registros. Apesar do número de registros considerado na totalidade, apenas 31 registros (27\% dos resgates) possibilitaram uma análise completa, inclusive espacial, devido aos dados incompletos dos formulários. Além disso, houve uma dificuldade de comparação com dados da literatura devido à escassez de estudos na área de resgate e soltura de animais silvestres.

Entre os grupos taxonômicos, o mais resgatado foi Mammalia, com 70 indivíduos $(61,4 \%)$, em seguida, Reptilia, com 34 indivíduos $(29,8 \%)$ e Aves, com 10 indivíduos (8,8\%) (Fig. 2; Tabela 1). 
Das espécies, a mais resgatada foi a preguiçacomum (Bradypus variegatus), com 37 indivíduos, representando $32,5 \%$ do total e $52,8 \%$ da classe Mammalia (Tabela 1). Devido à fragmentação ou destruição de seu habitat, é crescente a ocorrência de indivíduos dessa espécie no ambiente urbano (Pedrosa et al., 2016), o que pode ocasionar consequências negativas para esses animais, como atropelamento, choque elétrico e contrair patógenos (Pedrosa et al., 2016). A principal ameaça a esses animais arborícolas é a degradação e a fragmentação de habitat e seu deslocamento no solo, onde possuem pouca habilidade, os deixa expostos a diferentes ameaças, tais como fome, predadores e caça (Moreno \& Plese, 2006). Adicionalmente, indivíduos dessa espécie, que habitam os fragmentos cortados por estradas e rodovias, correm o risco em ser atingidos por automóveis ao tentar atravessá-las em direção à outra porção do fragmento (Pedrosa et al., 2016).
O fato de serem comumente resgatados por cidadãos ou autoridades e levados à sede do ICMBio Mamanguape pode ser explicado por serem mamíferos lentos quando se deslocam no solo, de fácil captura manual, e que apresentam atividade diurna, muitas vezes sendo facilmente vistos atravessando rodovias.

Entre os répteis, serpentes foi o grupo de maior representatividade, com 19 indivíduos (55,9\% dos répteis), em que se destacaram as espécies salamanta (Epicrates assisi), com 8 indivíduos, e jiboia (Boa constrictor), com 6 indivíduos, representando $42,1 \%$ e $31,6 \%$ das serpentes, respectivamente (Tabela 1). Estas são muitas vezes resgatadas pela equipe do ICMBio, por chamado da população, provavelmente por medo ou aversão de algumas pessoas. $\mathrm{O}$ grupo das aves foi o menos representativo, tanto em número de espécies como de indivíduos resgatados (Figura 2 ).

\section{Proporção de indivíduos resgatados por grupos taxonômicos}

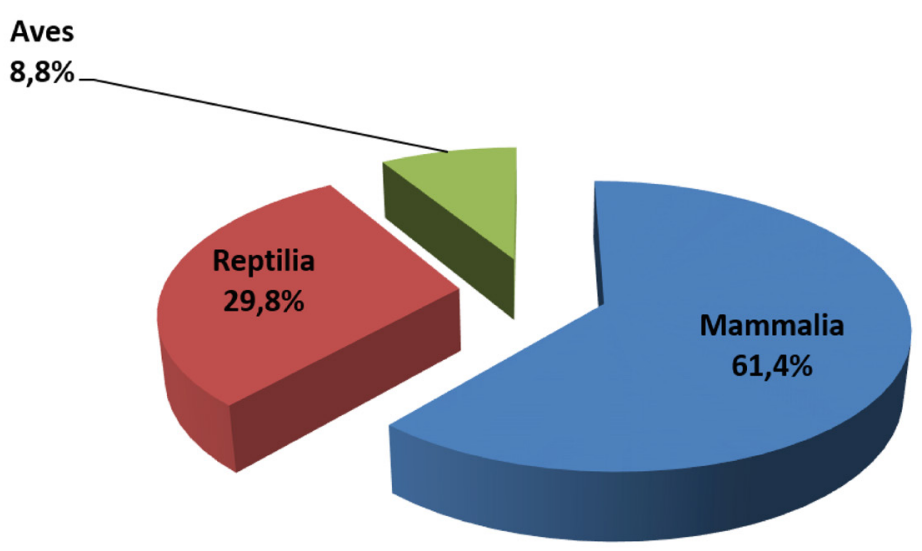

Figura 2 - Proporção de indivíduos resgatados, classificados por grupos taxonômicos.

Comparando-se esses dados com os de resgates feitos pelo Corpo de Bombeiros Militar de Guarabira (Alves, 2011), verificase a predominância da classe dos répteis, com 80,4\% (67,9\% destes, serpentes), em seguida de mamíferos, com $10,3 \%$ e aves, com 9,3\%.

A alta ocorrência de serpentes mostra alguma semelhança com o padrão encontrado neste estudo, com a principal diferença de, no caso da REBIO, o grande número de preguiçascomuns resgatadas diferir daquele de Alves (2011).
Essa maior proporção de serpentes resgatadas por bombeiros possivelmente se explica pelo fato de esses profissionais serem agentes de segurança pública e, portanto, mais acionados em casos de animais que geram insegurança à população. Cabe lembrar que não se pode comparar esses dados com os de estudos a partir de animais apreendidos, em que predominam as aves (Júnior et al., 2013; Silva, 2015), dada a diferença de motivos de sua chegada ao órgão ambiental. 
Tabela 1 - Lista de espécies de animais resgatados, com respectivo estado de conservação* e número de indivíduos.

\begin{tabular}{|c|c|c|c|}
\hline Nome científico & Nome vulgar & Estado de conservação & Indivíduos \\
\hline Aloutta belzebul & Guariba & VC & 6 \\
\hline Bradypus variegatus & Preguiça-comum & $\mathrm{NC}$ & 37 \\
\hline Callithrix jacchus & Sagui-de-tufo-branco & NC & 3 \\
\hline Cerdocyon thous & Raposa, cachorro-do-mato & NC & 3 \\
\hline Coendou prehensilis & Coendu, ouriço & NC & 6 \\
\hline Cyclopes didactylus & Tamanduaí & NC & 1 \\
\hline Dasyprocta iacki & Cutia & NC & 2 \\
\hline Dasypus novemcinctus & Tatu-verdadeiro, tatu-galinha & NC & 2 \\
\hline Didelphis albiventris & Timbu, gambá-de-orelha-branca & $\mathrm{NC}$ & 1 \\
\hline Euphractus sexcinctus & Tatu-peba & NC & 4 \\
\hline Galictis vittata & Furão & NC & 1 \\
\hline Puma yagouaroundi & Jaguarundi vermelho & VU & 1 \\
\hline Sylvilagus brasiliensis & Coelho selvagem, tapiti & NC & 1 \\
\hline Tamandua tetradactyla & Tamanduá-de-colete & NC & 2 \\
\hline Reptilia & & $\mathrm{NC}$ & \\
\hline Boa constrictor & Jiboia & NC & 6 \\
\hline Bothrops jararaca & Jararaca & NC & 1 \\
\hline Caiman latirostris & Jacaré-de-papo-amarelo & $\mathrm{NC}$ & 3 \\
\hline Chelonoidis carbonaria & Jabuti-piranga & NC & 3 \\
\hline Epicrates assisi & Salamanta, jiboia-arco-íris & NC & 8 \\
\hline Iguana iguana & Camaleão, iguana & NC & 4 \\
\hline Kinosternon scorpioides & Cágado, muçuã & NC & 2 \\
\hline Mabuya cf. macrorhyncha & Lagarto, briba & NC & 2 \\
\hline Micrurus sp & Cobra-coral & NC & 1 \\
\hline Oxyrhopus trigeminus & Cobra-coral-falsa & $\mathrm{NC}$ & 2 \\
\hline Philodryas nattereri & Cobra-corre-campo & NC & 1 \\
\hline Tupinambis sp & Teju & $\mathrm{NC}$ & 1 \\
\hline \multicolumn{4}{|l|}{ Aves } \\
\hline Athene cunicularia & Coruja buraqueira & $\mathrm{NC}$ & 1 \\
\hline Dendrocygna viduata & Marreco, irerê & NC & 1 \\
\hline Hydropsalis albicollis & Bacurau & $\mathrm{NC}$ & 2 \\
\hline Nothura maculosa & Codorna-amarela & NC & 1 \\
\hline Nyctibius griséus & Mãe-da-lua, urutau & NC & 1 \\
\hline Rupornis magnirostris & Gavião-carijó & $\mathrm{NC}$ & 3 \\
\hline Tyto albatuidara & Coruja-de-igreja & NC & 1 \\
\hline Total & & & 114 \\
\hline
\end{tabular}

*A sigla VU indica o estado de conservação vulnerável, de acordo com a Portaria MMA no 444/2014 (MMA, 2014), que reconhece a Lista Nacional Oficial de Espécies da Fauna Ameaçadas de Extinção e a sigla NC indica que a espécie não consta na referida lista.

Um dado que chama a atenção quanto à distribuição de solturas de Bradypus variegatus é sua concentração no setor SEMA 2 da REBIO Guaribas, especialmente na sede da unidade, havendo 13 registros, $38,23 \%$ do total de 34 solturas dessa espécie, no período considerado.
Todos os indivíduos soltos neste setor foram resgatados em seu limite imediato, ou na rodovia PB-71 ou na BR-101, que margeiam esta porção da reserva. Dentre as demais, 3 foram encaminhadas ao cativeiro e 21 foram soltas em outras localidades. 
Isso pode se explicar pelo fato de o setor SEMA 2 ser o maior fragmento de Mata Atlântica da Paraíba, bem como ele ser margeado pelas rodovias citadas, onde as preguiças são resgatadas e levadas para a atual sede do ICMBio Mamanguape.

No setor SEMA 3 da REBIO Guaribas, há problemas de viabilidade da população de preguiça-comum relacionados ao pequeno tamanho da população e à taxa reprodutiva das fêmeas (Pedrosa 2016). Como uma das formas de evitar a extinção dessa população em longo prazo, Pedrosa (2016) sugere translocar para seu interior machos adultos, um a cada quatro anos, reduzindo a desproporção sexual, consequentemente aumentando a probabilidade de encontros entre machos e fêmeas em períodos reprodutivos e elevando a taxa reprodutiva das fêmeas. Considerando a frequência elevada de resgates de indivíduos de preguiças-comuns que chegam à sede da REBIO, alguns desses indivíduos poderiam ser aproveitados em um programa de revigoramento populacional dessa espécie, ainda a ser criado. Dos registros de resgate de preguiça-comum em que foram assinalados os locais da soltura, apenas um, em 2012, foi feito nessa área, mas sem essa intenção. No caso de implementar esse programa, seria indicado realizar nova avaliação populacional, bem como o monitoramento dos indivíduos translocados em parceria com pesquisadores especializados.

Em relação ao tipo de resgatador, o cidadão e a equipe da REBIO tiveram quantidade de registros iguais, 52 resgates $(45,6 \%)$ cada um, e depois outras autoridades, com 10 resgates (8,8\%), sendo 1 pela Polícia Civil, 3 feitos pela Polícia Militar, 4, pela Polícia Rodoviária Federal, 1 pelo Corpo de Bombeiros e 1 pelo batalhão ambiental da PMPB (Fig. 3).

Uma parte considerável dos resgates feitos por cidadãos comuns provavelmente são representados por motoristas que passam pelas rodovias PB-071 e BR-101, e colaboram com o resgate de animais silvestres em risco de serem atropelados, especialmente indivíduos de preguiças-comuns. Dada a alta proporção de resgates feitos por cidadãos comuns, sugerimos uma revisão do conceito de resgate adotado pelo IBAMA na Instrução Normativa $n^{\circ} 23$ de 2014, uma vez que esta reconhece a autoria da atividade apenas por autoridades competentes (IBAMA, 2014).

\section{Proporção de eventos de resgate por tipo de resgatador}

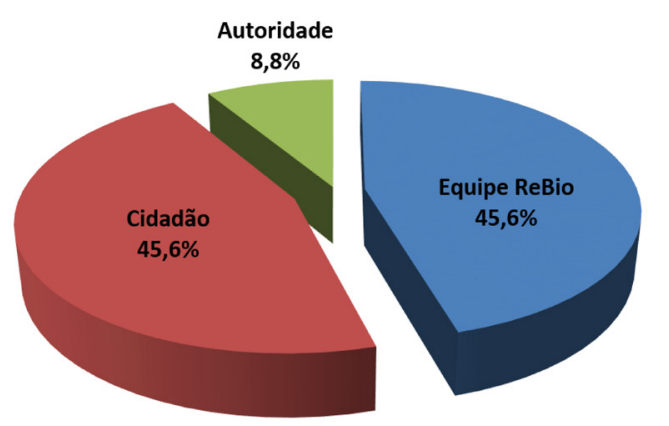

Figura 3 - Proporção de eventos de resgate, por tipo de resgatador.

Entre os tipos de destinação, dos 114 indivíduos, 87 foram destinados à soltura $(76,3 \%)$, 18 indivíduos ao cativeiro $(15,8 \%)$ e 9 indivíduos $(7,9 \%)$ destinados a coleção científica da Universidade Federal da Paraíba-Campus I(Fig. 4). Dessa forma, verifica-se que a maior demanda de resgates é para animais de aspecto $e$ comportamento saudável encontrados em locais de risco para eles mesmos ou para humanos.

\section{Proporção de indivíduos resgatados por grupos taxonômicos}

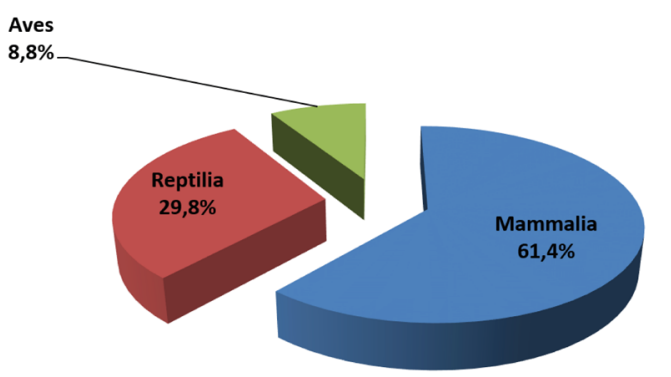

Figura 4 - Proporção de resgates, por tipo de destinação.

A elevada proporção de animais que vêm a óbito (Coleção, 7,9\%) e dos que são encaminhados a cuidados veterinários (Cativeiro, 15,8\%) demonstra um dano considerável que a fauna silvestre sofre nas imediações da UC. Presumimos que ele seja causado principalmente por atropelamentos nas rodovias e queimaduras nos plantios de canade-açúcar. Rosa \& Mauhs (2004) consideraram o atropelamento de animais um problema de menor destaque entre as questões que envolvem a ameaça das espécies da fauna brasileira. 
Mais recentemente, esse tipo de impacto adquiriu grande importância como preocupação para a conservação, de forma que uma grande quantidade de medidas de mitigação e dispositivos de engenharia podem ser usados para reduzir seus efeitos (Gomes et al., 2019).

Dos 87 registros de resgates com solturas, apenas foram obtidos 31 registros preenchidos com todas as informações necessárias para analisar espacialmente esses eventos. A partir desses, foi possível calcular um deslocamento médio de 546,4 metros para mais próximo da REBIO e uma distância média de 5.010 metros entre os pontos dos dois eventos. Desconsiderando-se um registro atípico, com resgate em Guarabira e soltura próxima à REBIO, obteve-se um deslocamento médio de 348,38 metros para mais distante da UC e uma distância média de 3891,39 metros entre os pontos dos dois eventos.

Os pontos de soltura encontram-se dispersos na região, porém, em alguns casos, eles concentraram-se muito próximos à sede administrativa da Reserva Biológica Guaribas, na SEMA 2 (Fig. 5). Isso é um sinal de que, em parte dos casos, os animais são levados para a sede $e$ lá mesmo sejam soltos por causa da praticidade. $\mathrm{O}$ fato de alguns animais serem encontrados atravessando as rodovias que margeiam a UC nos faz supor que eles a habitem por ela ser $\mathrm{o}$ fragmento mais próximo do encontro.

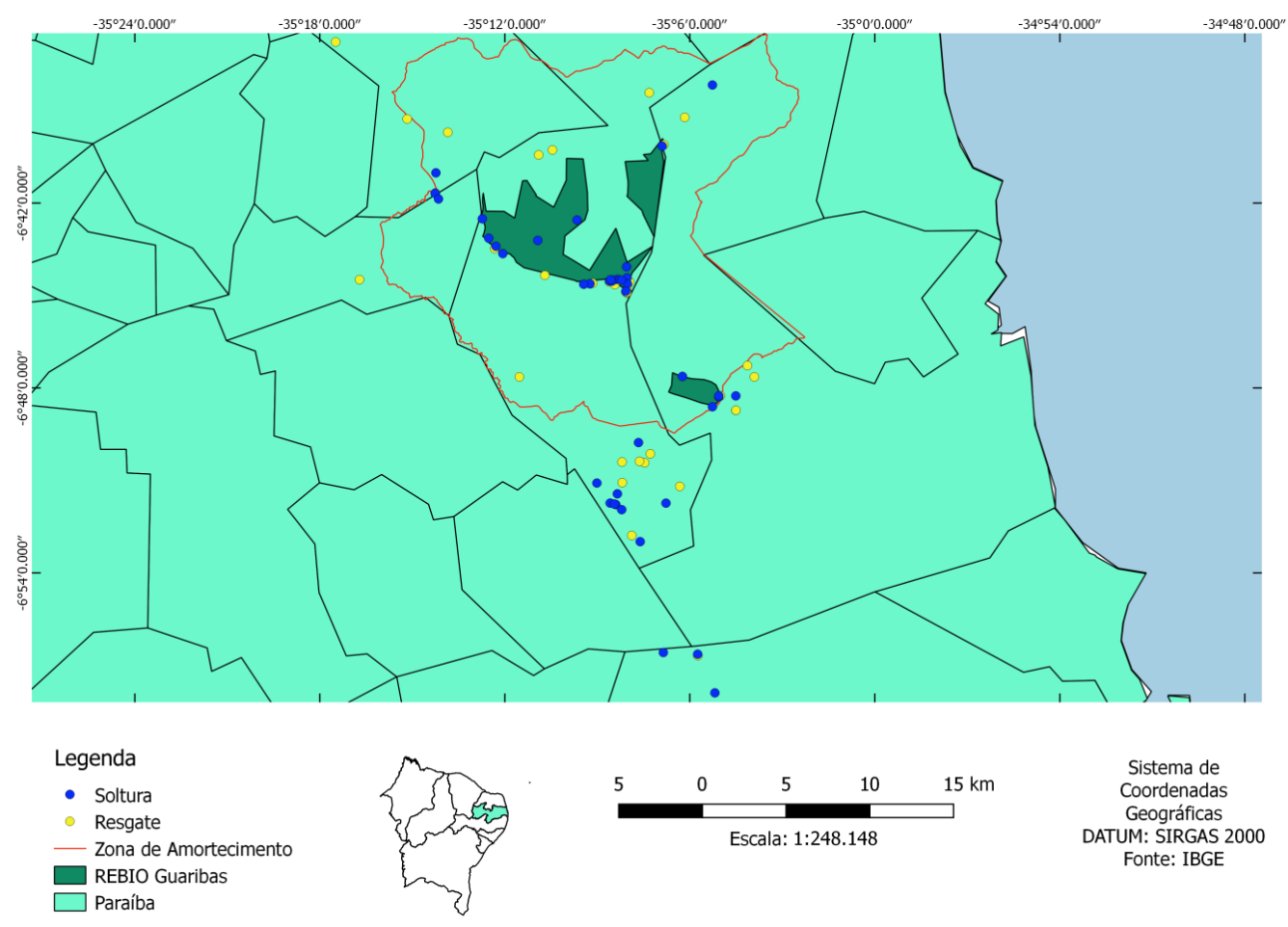

Figura 5 - Mapa indicativo dos resgates e solturas de fauna.

Dos 31 casos em que se teve o registro do ponto de resgate e o de soltura, pôde-se observar que o critério a ser adotado como de soltura em fragmentos florestais mais próximos do ponto de resgate foi obedecido em 21 casos $(67,7 \%)$ e não obedecido em 10 (32,3\%). Cabe lembrar que o fragmento florestal mais próximo com habitat adequado para a espécie resgatada nem sempre é o mais próximo de todos do local de resgate, o que pode contribuir para explicar parte dos resultados.

\section{Implicações para a conservação}

Antes de realizar as atividades de soltura, devem ser analisadas as áreas onde se planeja soltar os indivíduos, pois as solturas realizadas indevidamente podem comprometer o equilíbrio do ecossistema local. A ação mal planejada poderá acarretar um desequilíbrio ambiental, seja pelo aumento exagerado da população, excesso de competição por área de 
vida, introdução de uma espécie animal que não tenha ocorrência naquele local e introdução de novas doenças (Marini \& Marinho-Filho, 2006).

Segundo Branco \& Ribeiro (2011, p. $33)$, "os critérios adotados para recolocação dos animais resgatados na natureza exigem o conhecimento do meio, tanto das espécies que ocorrem na localidade como das doenças, além das condições ambientais". Fatores importantes e favoráveis para o retorno do animal é a identificação da espécie e a sua origem geográfica, ou seja, deve ser solto na área de procedência, pois pode ocorrer a introdução de uma espécie exótica. Esse é um risco que praticamente não se corre ao se praticar solturas a partir de resgates em áreas próximas, como é o caso em questão, pois os animais são capturados recém-saídos da natureza. Mas é um sério risco no caso de solturas a partir de apreensões, como reportado por Vidolin et al. (2004), pois, muitas vezes, os animais traficados são transportados para regiões distantes de sua área de origem.

Para se fazer solturas adequadas, devese avaliar o estado de saúde do animal, devido às doenças que podem ser transmitidas para indivíduos da mesma e de outras espécies. Como afirmam Marini \& Marinho-Filho (2011, p. 11), "tem que avaliar a capacidade de suporte do ambiente, pois além da definição do tipo de habitat apropriado, deve-se avaliar se a área de soltura ainda suporta mais indivíduos das espécies em questão". Um dos fatores essenciais para uma ação bem-sucedida seria o acompanhamento dos indivíduos soltos, para analisar se as atividades de soltura estão sendo eficazes. (Vidolin et al., 2004). A etapa final e uma das mais importantes, o monitoramento, é aquela capaz de confirmar se houve sucesso na atividade.

Infelizmente, esses procedimentos não são viáveis às autoridades que são demandadas a resgatar e receber animais resgatados e precisam tomar decisões rapidamente, inclusive a REBIO Guaribas, que pouco conta com parcerias para isso. A que já existe é a parceria intrainstitucional com o CPB/ICMBio que se aplica apenas ao Guariba (Allouata belzebul), deixando descobertas as demais espécies, inclusive ameaçadas de extinção, como o Jaguarundi (Puma yaguaroundi), cuja soltura de um indivíduo foi registrada neste trabalho.
O que se tem feito até o momento é apenas minimizar os riscos de prejuízos aos ecossistemas evitando solturas desordenadas, como eram feitas antes de 2010, concentrando-se todos os indivíduos na própria reserva. No entanto, o que a atual gestão do ICMBio Mamanguape poderia fazer era estabelecer uma parceria com instituição de pesquisa, a qual faria a análise clínica dos indivíduos resgatados, o monitoramento dos indivíduos soltos e decisões mais criteriosas do local de soltura. Além disso, deve-se investir na capacitação da equipe para melhorar a qualidade dos registros e na arregimentação de voluntários para maior apoio nessas tarefas.

\section{Conclusões}

Uma parte considerável da demanda de resgates da REBIO Guaribas destina-se à soltura, predominantemente de mamíferos e répteis, com destaque para a preguiça-comum, no primeiro grupo e as serpentes, no segundo. Há uma elevada proporção de indivíduos resgatados feridos a ponto de serem encaminhados a cuidados veterinários ou irem a óbito, cujas principais causas presumidas são atropelamentos em rodovias e queimaduras em canaviais. Detectou-se a oportunidade de aproveitar parte dos indivíduos de preguiçacomum resgatados e soltos na SEMA 2 em um programa de revigoramento populacional a ser criado na área da SEMA 3, detectada, em outro estudo, a alta probabilidade de extinção da espécie nesse fragmento florestal.

Não se pode concluirque as solturas tendem a aproximar ou afastar os animais resgatados da REBIO Guaribas, mesmo desconsiderando o registro discrepante. Embora a média de distanciamento dos 31 eventos resulte em um leve deslocamento para mais distante da reserva (348,38m), essa é baseada em 21 valores com tendência à aproximação, seis com tendência ao afastamento e quatro com deslocamento nulo. Isso significa que poucos eventos de afastamento de maior distância contribuíram para o resultando, diante de um número maior de eventos de aproximação. A soltura no fragmento florestal mais próximo do ponto de resgate foi realizada em, aproximadamente, duas vezes o número de casos analisados em que ela não foi. Parte dos últimos casos podem ser explicados pela busca por fragmentos com o habitat adequado para a espécie resgatada. 
A equipe da UC teria condições de melhorar a qualidade dos registros $e$ atender melhor os critérios já estabelecidos, por meio da capacitação dos principais executores dos resgates e solturas, que são servidores com perfil operacional e brigadistas temporários, bem como com a incorporação de voluntários. Mas devese ter em vista que a qualidade da informação sempre terá uma limitação imposta pelo fato de o cidadão comum ser o resgatador mais frequente, empatado com a equipe da UC e, muitas vezes, este não fornece informações completas.

Embora não seja atribuição da equipe de uma unidade de conservação realizar ou receber resgates de fauna, ela acaba atuando como um centro de resgate de fauna por ser a única opção que a população das redondezas tem. Além disso, conforme nossa experiência, o próprio CETAS do IBAMA não tem condições de receber tantos animais, além de seus agentes não terem como se deslocar para tão distante da capital do estado. Portanto faz-se necessária uma parceria com instituição com condições de oferecer apoio para decisões de destinação mais qualificadas $e$ monitoramento das ações de manejo, apoiada pela logística de campo fornecida pelo ICMBio Mamanguape.

\section{Agradecimentos}

Agradecemos a Murilo Abacherli de Camargo (UFPB), ao editor Carlos Abrahão $e$ a três revisores anônimos por comentários $e$ correções a versões prévias deste artigo. Esta pesquisa foi autorizada pelo ICMBio por meio da autorização SISBIO no 56923-1. Uma bolsa do Centro de Integração Empresa-Escola (CIEE) foi concedida a TBS durante parte de seu estágio de iniciação científica.

\section{Referências}

Alves IBS. Diversidade do resgate de fauna e as ações do $3^{\circ}$ batalhão de Bombeiros Militar da Paraíba em Guarabira-PB. Monografia (Licenciatura Plena em Ciências Biológicas). Universidade Estadual da Paraíba. 65p. 2011.

Branco AM. Centro de Manejo de Animais Silvestres, p. 235-253. In: Giovanini D. (org.). Animais silvestres: vida à venda. Editora Dupligráfica 260p. 2002.
Branco AM \& Ribeiro H. Descentralização da gestão e manejo da fauna silvestre: o caso da divisão técnica de medicina veterinária e manejo da fauna silvestre do município de São Paulo. Interfacehs. 6(1): 20-38, 2011.

Dias-Júnior MBF, Cunha HFA \& Dias TCAC. Análise da destinação da fauna silvestre apreendida no Estado do Amapá, Brasil. Planeta Amazônia: Revista Internacional de Direito Ambiental e Políticas Públicas. 5: 23-36, 2013.

Equipe de Desenvolvimento do QGIS. Sistema de Informação Geográfica QGIS, versão 2.10. Projeto da Fundação Geoespacial de Código Aberto. < http://qgis. osgeo.org > . Acesso em: 16/05/2016.

Gomes LP, Caixeta FF, Torres RR, Castro DN, Thomé GA, Lima, RIP, Sousa ACP, França RM \& Silva LL. Fauna run-over mitigation measures on brazilian federal highway concessions. Heringeriana, 13(1): 1020, 2019.

IBAMA (Instituto Brasileiro do Meio Ambiente e dos Recursos Naturais Renováveis), 2003. Plano de Manejo da Reserva Biológica Guaribas, 520p.

IBAMA (Instituto Brasileiro do Meio Ambiente e dos Recursos Naturais Renováveis). Instrução Normativa n²3 de 31 de dezembro de 2014. Diário Oficial da União, Seção 1: 115-118, 2014.

IUCN/SS. Guidelines for Reintroductions and Other Conservation Translocations. Version 1.0. Gland, Switzerland: IUCN Species Survival Commission. 57 p. 2013.

Marini MA \& Marinho-Filho JS. Translocação de Aves e Mamíferos: Teoria e Prática no Brasil, p. 505-536. In: Rocha CFD, Bergallo HG, Van-Sluys M. \& Alves MAS (orgs.). Biologia da conservação: essências. Editora Rima. 582p. 2006.

MMA (Ministério do Meio Ambiente). Portaria N ${ }^{\circ} 444$, de 17 de dezembro de 2014. Diário Oficial da União, Seção 1, 245: 121-126, 2014.

Moreno S \& Plese T. The illegal traffic in sloths and threats to their survival in Colombia. Edentata,7: 10$18,2006$.

Pedrosa EP. Análise da viabilidade populacional da preguiça-comum (Bradypus variegatus Schinz, 1985) em fragmento de mata atlântica $e$ as principais ameaças dos ambientes urbanos na Paraíba. Dissertação (Mestrado em Ecologia e Monitoramento Ambiental). Universidade Federal da Paraíba. 93p. 2016. 
Pedrosa EP, França RC \& Castro CSS. Mudanças na paisagem e impactos da área urbana no comportamento da preguiça-comum (Bradypus variegatus Schinz, 1825) na APA da Barra do Rio Mamanguape, Paraíba, Brasil, p. 195-218. In: Almeida, N.V. \& Silva, M.D. (orgs). Geotecnologia e Meio Ambiente: Analisando uma área de proteção ambiental. Editora FeF. 238p. 2016.

Rocha-Mendes F, Napoli RP \& Mikich SB. Manejo, reabilitação e soltura de mamíferos selvagens. Arquivos de Ciências Veterinárias e Zoologia, 9(2): 105-109, 2006.

Rosa AO, \&Mauhs J. Atropelamento de animais silvestres na rodovia RS-040. Caderno de Pesquisa Série Biologia, 16 (1): 35-42, 2004.

Silva NS. Espécimes recebidos no centro de triagem de animais silvestres de Salvador/BA durante os anos de 2012 a 2014. Monografia (Graduação em Medicina Veterinária. Salvador, BA: Universidade Federal da Bahia. 47p. 2015.
Valença-Montenegro $\mathrm{M}$, Martins $\mathrm{AB}$, Laroque $\mathrm{PO}$, Azevedo RB, Buss G \& Ludwig G. Histórico e perspectivas da reintrodução do guariba-de-mãosruivas (Alouatta belzebul) na Reserva Biológica que leva seu nome. p. 51-53. In: Leal, AH. (Ed.) Memórias da Reunião de Pesquisa e Conservação da Natureza no Litoral Norte da Paraíba: manejando a pesquisa e pesquisando o manejo das áreas protegidas. Mamanguape, ICMBio: 2018. Disponível em: < https:// www.icmbio.gov.br/portal/images/stories/Leal_2018 Mem\%C3\%B3rias_da_RPCN_Litoral_Norte_PB_2.pdf $>$. Acesso em21/09/2020.

Vidolin GP, Mangini, PR, Moura-Britto M. \& Muchailh, MC. Programa Estadual de Manejo de Fauna Silvestre Apreendida - Estado do Paraná, Brasil. Cadernos da Biodiversidade, 4(2): 37-49, 2004.

\section{Biodiversidade Brasileira - BioBrasil. \\ Edição Temática: PIBIC}

$$
\text { n. 1, } 2022
$$

http://www.icmbio.gov.br/revistaeletronica/index.php/BioBR

Biodiversidade Brasileira é uma publicação eletrônica científica do Instituto Chico Mendes de

Conservação da Biodiversidade (ICMBio) que tem como objetivo fomentar a discussão e a disseminação de experiências em conservação e manejo, com foco em unidades de conservação e espécies ameaçadas. 